Kurzvortrag - 6. Scenario Forum Symposium

\title{
Mimetische Kommunikation
}

\section{Edith Karimi}

Im Fokus meines Forschungsinteresses und meiner Arbeit stehen Sprachbildung und -förderung durch ästhetische Erfahrung. Meine Forschung und meine Arbeit in literarischen Kunstprojekten mit Kindern haben mich davon überzeugt, dass Sprache in mimetischen Prozessen erworben wird. Die Verständigung wurzelt in mimetischer Begabung des Menschen (vgl. Gebauer \& Wulf 2003; Karimi 2016).

Mit dem Bildungswissenschaftler Alex Baumgartner verstehe ich Erfahrung „als ein Sich-Einlassen auf das zu begreifende Objekt“ (Baumgartner 1999: 195). Ästhetische Erfahrung wird durch die Voraussetzungen unseres Wahrnehmens konstituiert. Sich auf einen Gegenstand ästhetisch einzulassen, um ihn zu begreifen, setzt nämlich voraus, dass er leiblich und sinnlich erkannt wird. Eine ästhetische Erfahrung ist eine sinnlich-sinnhafte und emotionale Erfahrung. Verankert ist sie im mimetischen Gestaltungsvermögen des Menschen an sich selbst und an der Welt (vgl. Mollenhauer 1999; Baumgartner 1999; Wulf et al. 2005, 2007, 2014a).

Wulf führt dazu aus: „Die mimetische Begegnung mit der Welt erfolgt mit allen Sinnen, die im Verlauf dieser Prozesse ihre Sensibilität entfalten“ (Wulf 2007: 95). Dabei wird der menschliche Körper zum Ort der erlebten Bilder-, Klangkörper-, Tast-, Geruchs- und Geschmackswelten (vgl. Wulf et al. 1999, 2005, 2007, 2014a, 2014b).

Drei Voraussetzungen gelten, so der deutsche Anthropologe, wenn von Mimesis gesprochen wird (vgl. Wulf 2014b: 247f):

1. Mimesis ist ein zentraler Begriff der Ästhetik, in seinem ursprünglichen Sinn vor allem als die Nachahmung der Natur durch die Kunst verstanden.

2. Im Kontext der Kulturen des Performativen komme der sprachgeschichtliche Ursprung des Begriffs „Mimesis“ zum Tragen. Er verweist auf den performativen Charakter mimetischer Handlungen.

3. Mimesis ist ein anthropologischer Begriff. In anthropologischer Hinsicht steht außer Zweifel, dass menschliche Entwicklung und Bildung ohne die besondere Fähigkeit zu mimetischem Handeln nicht möglich ist. Die Fähigkeit zur Mimesis ist also eine conditio humana, eine anthropologische Bedingung des Menschen.

Der Mensch bemüht sich von Anfang an um eine kreative Ähnlichkeitsbeziehung zum Außen, insbesondere zu anderen Menschen. Seine mimetischen 
Bezugnahmen sind dabei sowohl reproduktiv als auch kreativ und enthalten sowohl passive als auch aktive Elemente (vgl. Gebauer \& Wulf 2003; Wulf 2014b).

Der mimetische Bildungsprozess vollzieht sich im Spannungsbogen der Dialektik aus Wiederholung und Variation, Nachahmung und Schöpfung, Identifikation und Distanzierung, aus Nähe und Eigensinn, Ähnlichkeit und Autonomie, aus Übereinstimmung und Unbestimmtheit (vgl. auch Ehlers 1997).

Der Mensch bildet sich durch Mimesis, und dieser Bildungsprozess hat eine sinnlich-ästhetische Dimension. Bildung ist an mannigfaltige, differenzierte Selbst- und Weltbezüge angewiesen (Humboldt), die in ganzheitlichen Erlebniszusammenhängen, im Einklang von Sinnlichkeit, Emotionalität und Vernunft, den Menschen in seiner Leib-Seele-Einheit einbeziehen und seine Handlungsmöglichkeiten erweitern (vgl. Baumgartner 1999).

Die Sprache ist zuallererst ein körperlich und sinnlich spürbares Phänomen, das mit konkreten sinnlich erlebten Situationen verbunden ist. Mollenhauer zufolge wird in der Sprache die äußere und innere Sinnlichkeit repräsentiert (vgl. Mollenhauer 1999: 91f).

Auf sie ist mimetisch der Darstellungs-, Referenz- und Deutungsaspekt der Sprache in Kommunikationsakten bezogen. Fischer-Lichte betonnt die konstitutive Rolle der ästhetischen Erfahrung für die Entstehung von Bedeutung: „Im Prozeß der ästhetischen Erfahrung tritt so besonders plastisch hervor, daß Bedeutung ohne den Körper nicht zu denken und zu haben ist, daß sich bei Bedeutung in diesem Sinne immer um verkörperte Bedeutung handelt" (FischerLichte 2001: 356). Das Leiberlebnis schafft eine unmittelbare Verbindung von Bedeutung mit Körperbewegung, Gebärden, Stimme, Atem, Mimik, Gestik, Klängen, Raum, Licht, Wärme etc.

Der berühmte Sprachphilosoph Wilhelm von Humboldt gelangte in umfassenden Sprachstudien zu der Erkenntnis, dass die Sprache menschliche Tätigkeit an der Welt sei (vgl. Humboldt zit. nach Benner 1990: 136f).

In dieser performativen Betrachtungsweise der Sprache geht es um das Wie im Prozess des Repräsentierens und des Umgangs mit den Repräsentationen, es geht um die Dramaturgie der Sprach- und Handlungsvollzüge und um die Körperlichkeit und Materialität von Kommunikationsprozessen (vgl. Wulf et al. 2005, 2017; Wulf \& Zirfas 2007).

Dieses Verständnis der Sprache impliziert, dass eine gemeinsame Sprache miteinander zu sprechen, gemeinsam tätig zu sein, heißt. Gemeinsam vollzogene Handlungen bringen gemeinsam geteilte Wirklichkeit hervor (vgl. Zirfas 2017).

Wenn eine Person zu einer anderen spricht, führt sie ihre Denktätigkeit mit den ihr verfügbaren angeborenen spontanen biologischen und soziokulturellen Kommunikationsformen auf. Sie spricht in verbaler und nonverbaler Sprache mit Worten, Melodie, Blickkontakt, Gesichtsausdruck, Gestik, Bildern, Requisiten, Schrift, Körperhaltung und -bewegungen verschiedenster Art.

Sie musst das tun, weil nur das, was der Adressat oder die Adressatin ihrer Äußerung sinnlich wahrnehmen kann, kann er oder sie es mit der Ganzheit von Leib und Seele, Gefühl und Vernunft aufgreifen und es imaginär oder konkret 
materiell handelnd nachvollziehen, das heißt nachahmen.

Sich miteinanderzu verständigen, beinhaltetzugleich auch, dem Gesprächspartner/der Gesprächspartnerin das aus der Äußerung hervorgehende gemeinsame Handeln zu ermöglichen. In subjektiver Perspektivübernahme geht es also darum, sich in ihn oder sie hineinzuversetzen und Korrespondenzen zu suchen, zu wählen, zu äußern, die das gemeinsame nachahmende Tun in Bewegung setzen und in Bewegung halten.

Weil die Sprache aber nicht identisch mit dem Leben und mit dem Denken werden kann (vgl. Wygotski 1971; Papadopoulos 2010; Keiler 2002; Benner 1990), der Ausdruck also mehrdeutig ist, oder wie die Schöpfungen der Kunst einen Bedeutungsüberschuss besitzt, und weil auch die Simulation der Innenwelt des Gesprächspartners/der Gesprächspartnerin immer einen spekulativen und hypothetischen Charakter hat, sind Korrekturen, Verbesserungen, Abstimmungen im Verständigungsprozess unerlässlich, die von der Einsicht begleitet werden sollten, wie sie auch Wilhelm von Humboldt (vgl. Wygotski 1976: 47) formuliert, dass zu jedem Verstehen das Nichtverstehen gehöre.

Der Mensch kommuniziert in mimetischen Bezügen auf Vorgängiges, Erinnertes, Erfahrenes, auf die aktuellen Gedanken, auf die Gesprächspartner und Zuhörer, auf den Raum und seine Gegenstände und ihre Anordnung. Und jedes Mal, wenn er spricht, erschafft er etwas Eigenes und Gemeinsames mit neuer Qualität. Somit setzt der zwischenmenschliche Dialog die performative Kraft der Sprache frei, die u. a. mit Kreativität, tiefem Lernen, Verwandlung, Transformation, Reformation, Reflexion, Offenheit einhergeht.

Unser Dialog über den Weg zu einer neuen, performativen Lehr- und Lernkultur während des 6th SCENARIO Forum Symposiums mündet für mich in der Erkenntnis: Wenn gelungene Bildung an Bedingungen zur Konstitution autonomer und zugleich sozial verantwortungsvoller Handlungsfähigkeit und Subjektivität gebunden ist, dann ist sie in ästhetischen Erfahrungen und insbesondere in der Kunst zu verankern.

\section{Bibliografie}

Baumgartner, Alex (1999): Die Dialektik von Bildung und Bildern. In: Schäfer, Gerd \& Wulf, Christoph (Hrsg.): Bild - Bilder - Bildung. Weinheim: BELTZ, 195-226

Benner, Dietrich (1990): Wilhelm von Humboldts Bildungstheorie. Eine problemgeschichtliche Studie zum Begründungszusammenhang neuzeitlicher Bildungsreform. Weinheim, München: Juventa

Ehlers, Katrin (1997): Mimesis und Theatralität. Dramatische Reflexionen des modernen Theaters im 'Theater auf dem Theater'. Münster et al.: Waxmann

Fischer-Lichte, Erika (2001): Ästhetische Erfahrung. Das Semiotische und das Performative. Tübingen: Francke 
Gebauer, Gunter \& Wulf, Christoph (2003): Mimetische Weltzugänge. Soziales Handeln - Rituale und Spiele - ästhetische Produktionen. Stuttgart: Kohlhammer

Karimi, Edith (2016): Mimetische Bildung durch Märchen. Phantasie, Narration, Moral. In: Wulf, Christoph (Hrsg.): European Studies in Education. Volume 34. Münster: Waxmann

Keiler, Peter (2002): Lev Vygotskij - ein Leben für die Psychologie. Weinheim, Basel: Beltz

Mollenhauer, Klaus (1999): Ästhetische Erfahrung von Kindern beim Textschreiben. Ein Diskussionsbeitrag. In: Neuß, Norbert (Hrsg.): Ästhetik der Kinder. Interdisziplinäre Beiträge zur ästhetischen Erfahrung von Kindern. Frankfurt am Main: Gemeinschaftswerk der Evangelischen Publizistik e.V., Abt. Verlag, 83-94

Papadopoulos, Dimitris (2010): L.S. Wygotski. Werk und Rezeption. 2. Aufl. Berlin: Lehmanns Media

Wulf, Christoph (1999): Bild und Phantasie. Zur historischen Anthropologie des Bildes. In: Schäfer, Gerd \& Wulf, Christoph (Hrsg.): Bild - Bilder Bildung. Weinheim: Beltz/Deutscher Studien Verlag

Wulf, Christoph (2017): Mimesis, Mimikry, Mimese. In: Ullrich, Jessica \& Ulrich Antonia (Hrsg.): Tierstudien (11/2017). Berlin: Neofelis, 13-21

Wulf, Christoph (2014a): Bilder des Menschen. Imaginäre und performative Grundlagen der Kultur. Bielefeld: transcript

Wulf, Christoph (2014b): Mimesis. In: Wulf, Christoph \& Zirfas, Jörg (Hrsg.): Handbuch Pädagogische Anthropologie. Wiesbaden: Springer VS, 247-257

Wulf, Christoph (2007): Mimetisches Lernen. In: Göhlich, Michael; Wulf, Christoph \& Zirfas, Jörg (Hrsg.): Pädagogische Theorien des Lernens. Weinheim, Basel: Beltz, 91-101

Wulf, Christoph (2005): Zur Genese des Sozialen. Mimesis, Performativität, Ritual. Bielefeld: transcript

Wulf, Christoph \& Zirfas, Jörg (2007): Performative Pädagogik und performative Bildungstheorien. Ein neuer Fokus erziehungswissenschaftlicher Forschung. In: Wulf, Christoph \& Zirfas, Jörg (Hrsg.): Pädagogik des Performativen: Theorien, Methoden, Perspektiven. Weinheim, Basel: Beltz, 7-12

Wygotski, Lew Semjonowitsch (1971): Denken und Sprechen. Mit einer Einleitung von Th. Luckmann, hrsg. v. J. Helm. Stuttgart: S. Fischer.

Wygotski, Lew Semjonowitsch (1976): Psychologie der Kunst. Dresden: VEB Verlag der Kunst

Zirfas, Jörg (2017): Zur Performativität der Pädagogik. In: Hudelist, Andreas \& Krammer, Stefan (Hrsg.): Kultur des Performativen 3/41. Innsbruck: Studien Verlag, 18-29 